DOE/PU/94217-DTS

DOE/MST-9504

\title{
A STUDY OF \\ MULTISTAGE/MULTIFUNCTION COLUMN FOR FINE PARTICLE SEPARATION
}

\section{QUARTERLY TECHNICAL PROGRESS REPORT}

(July 1, 1995 - September 30, 1995)

Principal Investigator: Dr. Shiao-Hung Chiang

Project Officer:

Dr. Ralph W. Lai

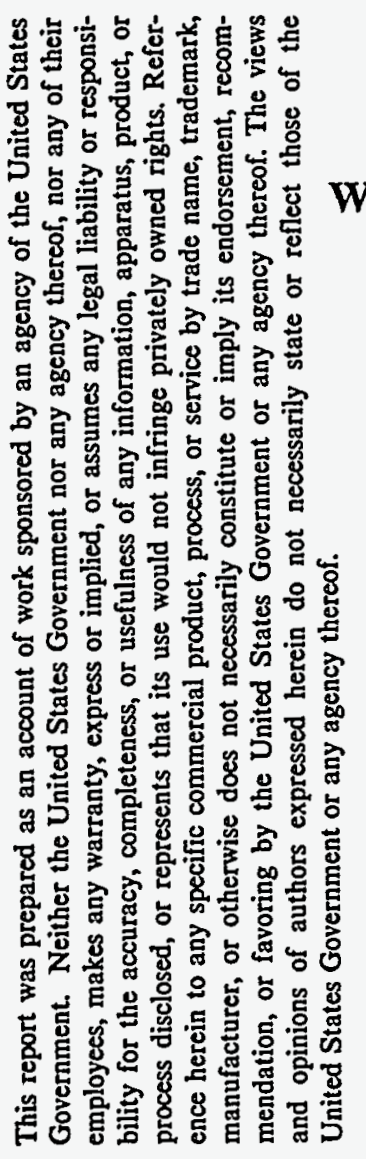

Work Performed under DOE Contract No. DE-PG22-94PC94217

(Award: \$200,000; Duration: July 8, 1994 - July 7, 1997)

Submitted to

U.S. Department of Energy

Pittsburgh Energy Technology Center

Pittsburgh, PA 15236-0940

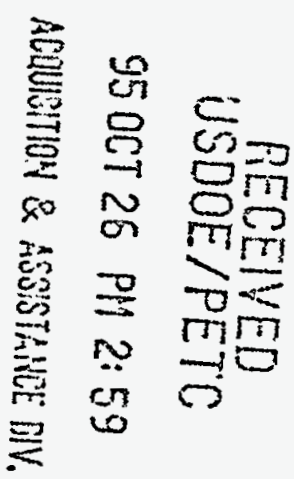

by

Chemical and Petroleum Engineering Department

University of Pittsburgh

Pittsburgh, PA 15261

October 20, 1995 


\subsection{INTRODUCTION}

The overall objective of the proposed research program is to explore the potential application of a new invention involving a multistage column equipped with vortex-inducing loopflow contactors (hereafter referred to as the multistage column) for fine coal cleaning process. The research work will identify the design parameters and their effects on the performance of the separation process. The results of this study will provide an engineering basis for further development of this technology in coal cleaning and in the general areas of fluid/particle separation.

In the last quarter, we performed image analyses of gas bubble size using photographs obtained from the multistage column ${ }^{[1]}$. In this quarter, the bubble size measurements in the conventional column was carried out. Also, correlations for results on gas holdup, bubble size and specific interfacial area were developed. In Table 1.1, the project schedule shows work accomplished to data.

Table 1.1 Project Schedule

\begin{tabular}{|c|c|c|c|c|c|c|c|c|c|c|c|c|c|}
\hline \multirow{2}{*}{ Tasks } & \multicolumn{3}{|c|}{1994} & \multicolumn{3}{|c|}{1995} & \multicolumn{3}{|c|}{1996} & \multicolumn{4}{|c|}{1997} \\
\hline & 7 & 9 & 12 & 3 & 6 & 9 & 12 & 3 & 6 & 9 & 12 & 3 & 6 \\
\hline 1. Project Planning & 网 & & & & & & & & & & & & \\
\hline 2. Equipment & & {$[x$} & $x$ & & & & & & & & & & \\
\hline 3. Hydrodynamic Tests & & & & & & & ב & & & & & & \\
\hline 4. Separation Tests & & & & & & & 口 & & & & & & $\sqsupset$ \\
\hline 5. Conventional Column Tests & & & & & & & & & & & & & \\
\hline 6. Data Analysis & & & & & & 正 & & & & & & 7 & ב \\
\hline 7. Reports & & 口 & $\square$ & $\square$ & 图 & $\square$ & $\square$ & $\square$ & 图 & ㅁ & $\square$ & $\square$ & \\
\hline
\end{tabular}

Notes: 口 Quarterly Technical Progress Report; 0 Annual Report; $\odot$ Final Report. 


\subsection{TECHNICAL PROGRESS}

\subsection{Task 1: Project Planning}

This task was completed in September $1994^{[1]}$.

\subsection{Task 2: Equipment Design and Construction}

Task 2 was completed in March $1995^{[2]}$.

\subsection{Task 3: Hydrodynamic Tests}

The objective of this task is to pursue a basic understanding of the hydrodynamic behavior and to characterize the flow and mixing conditions in a multistage separation column. In the last quarter, we have completed the measurements of bubble sizes in the three-stage column. During this quarter, we continued to perform image analyses of the bubble sizes based on the test data. The results are listed in the Appendix. The average size for the bubbles in the multistage column was found to range from $0.001 \mathrm{~m}$ to $0.002 \mathrm{~m}$. In September 1995, we began to work on correlation of data on gas holdup, bubble size and specific interfacial area obtained from the three-stage column and conventional column. Much of the current work has focused on developing a mathematical method to correlate the experimentally measured data in terms of operating parameters. (See Task 6 below).

\subsection{Task 4: Separation Tests}

This task will not be initiated until January 1996. 


\subsection{Task 5: Conventional Tests}

Similar experiments as that of Task 3 have been carried out to study the holdup, bubble size and specific interfacial area in the conventional flotation column. The results are analyzed and discussed in the next section.

\subsection{Task 6: Data Analysis}

For engineering design and scale-up, an understanding of the hydrodynamic behavior of the multistage column is required. Gas holdup $(\Phi)$ and specific interfacial area $(a)$ are two key parameters for evaluating the performance of the multistage column. For these purposes, gas holdup and bubble size $\left(\mathrm{d}_{\mathrm{av}}\right)$ as well as specific interfacial area data were experimentally determined (Tasks 3 and 5).

These experimental data ${ }^{[3-4]}$ are analyzed in this section in terms of three key operating parameters: superficial gas velocity $(V)$, agitation speed $(N)$ and frother dosage $(C)$. A secondorder polynomial equation ${ }^{[5]}$ is used for the correlation:

$$
Y=\beta_{0}+\sum_{i=1}^{k} \beta_{t} X_{1}+\sum_{i=1}^{k} \beta_{u} X_{i}^{2}+\sum_{i=1}^{k} \sum_{j>i}^{k} \beta_{i j} X_{t} X_{j}
$$

where, $Y$ represents $\Phi, \mathrm{d}_{\mathrm{av}}$ or $a ; X_{i}^{\prime}$ 's represent operating parameters, i.e., $X_{1}=C ; X_{2}=N ; X_{3}$ $=\mathrm{V}$; and $\beta_{o}, \beta_{i}, \beta_{i i}, \beta_{i j}$, are regression coefficients. The following is a summary of the correlations for these parameters. 


\subsubsection{Gas Holdup}

Gas hold-up is one of the key parameters for evaluating the performance of the multistage column. Based on Eq. (2.6.1) and using a multi-parameter regression program ${ }^{[6]}$, a correlation for the overall gas holdup in the multistage column can be obtained as follows:

$$
\begin{aligned}
\Phi= & -0.122+7.88 \times 10^{-3} \mathrm{C}+3.55 \times 10^{-3} \mathrm{~N}+6.11 \mathrm{~V}-2.13 \times 10^{-4} \mathrm{C}^{2}-4.37 \times 10^{-5} \mathrm{~N}^{2} \\
& -34.7 \mathrm{~V}^{2}+5.61 \times 10^{-5} \mathrm{CN}+9.32 \times 10^{-2} \mathrm{CV}+9.70 \times 10^{-3} \mathrm{NV}
\end{aligned}
$$

Figure 2.6.1 presents a comparison of the overall gas holdup between calculated values and experimental data. The relative deviation shown in the Figure 2.6 .1 is about $\pm 15 \%$.

\subsubsection{Bubble Size}

The bubble sizes in the multistage column were investigated using photographic method. The experimental setup consisted of a camera, a strobe light, and a video camera recorder. In order to save time in counting and sizing of a large number of gas bubbles, information concerning the size and shape of gas bubbles was obtained from the photographs with the aid of a computerized image analyzer. A software package for image analysis (HARMONY) ${ }^{[7]}$ was used to examine the bubble images. The Sauter mean diameter, $d_{a v}$, refers to the diameter of an equivalent sphere with the same volume-to-surface ratio as the gas bubble. For a distribution of bubbles, the Sauter mean diameter is defined as

$$
d_{a v}=\frac{\sum_{i=1}^{n} n_{i} d_{t}^{3}}{\sum_{i=1}^{n} n_{i} d_{i}^{2}}
$$


where $n_{i}$ denotes the frequency (numbers of bubbles over a specific size range) of appearance of bubbles with diameter $d_{i}$.

In order to calculate the $d_{a v}$ of the bubbles, more than 100 bubbles were counted for each run using the image analyzer. The Sauter mean diameter of bubbles in the multistage column was found to range from $0.001 \mathrm{~m}$ to $0.002 \mathrm{~m}$ which is comparable to the bubble sizes in the convention agitated column. However, in terms of bubble size, the design configuration of multistage column has a significant advantage over the conventional column. First, the multiple draft tubes are able to create an even distribution of bubbles over the entire column cross-section area, leading to almost no "dead" volume for bubble trajectories. In the conventional column, bubbles only appear in the area surrounding the shaft due to centripetal force, resulting in a low gas holdup and a poor dispersion condition. Second, even though the bubble sizes are comparable in both column configurations, the bubble sizes in multistage column are more uniform than those in the conventional column. Under this situation, the gas holdup in the multistage column are $20-50 \%$ higher than that in the conventional column. Thus, the multistage column provides a larger interfacial area than the conventional column.

Based on Eq. (2.6.1) and using the multi-parameter regression program, the correlation for the Sauter mean diameter of bubbles in the multistage column can be obtained as follows:

$$
\begin{aligned}
\mathrm{d}_{\mathrm{av}}= & 2.82 \times 10^{-3}-1.01 \times 10^{-4} \mathrm{C}-3.47 \times 10^{-5} \mathrm{~N}-1.34 \times 10^{-2} \mathrm{~V}+2.97 \times 10^{-6} \mathrm{C}^{2}- \\
& 1.84 \times 10^{-7} \mathrm{~N}^{2}+0.54 \mathrm{~V}^{2}-3.09 \times 10^{-7} \mathrm{C} \mathrm{N}-8.0 \times 10^{-4} \mathrm{C} \mathrm{V}+5.66 \times 10^{-4} \mathrm{~N} \mathrm{~V}
\end{aligned}
$$




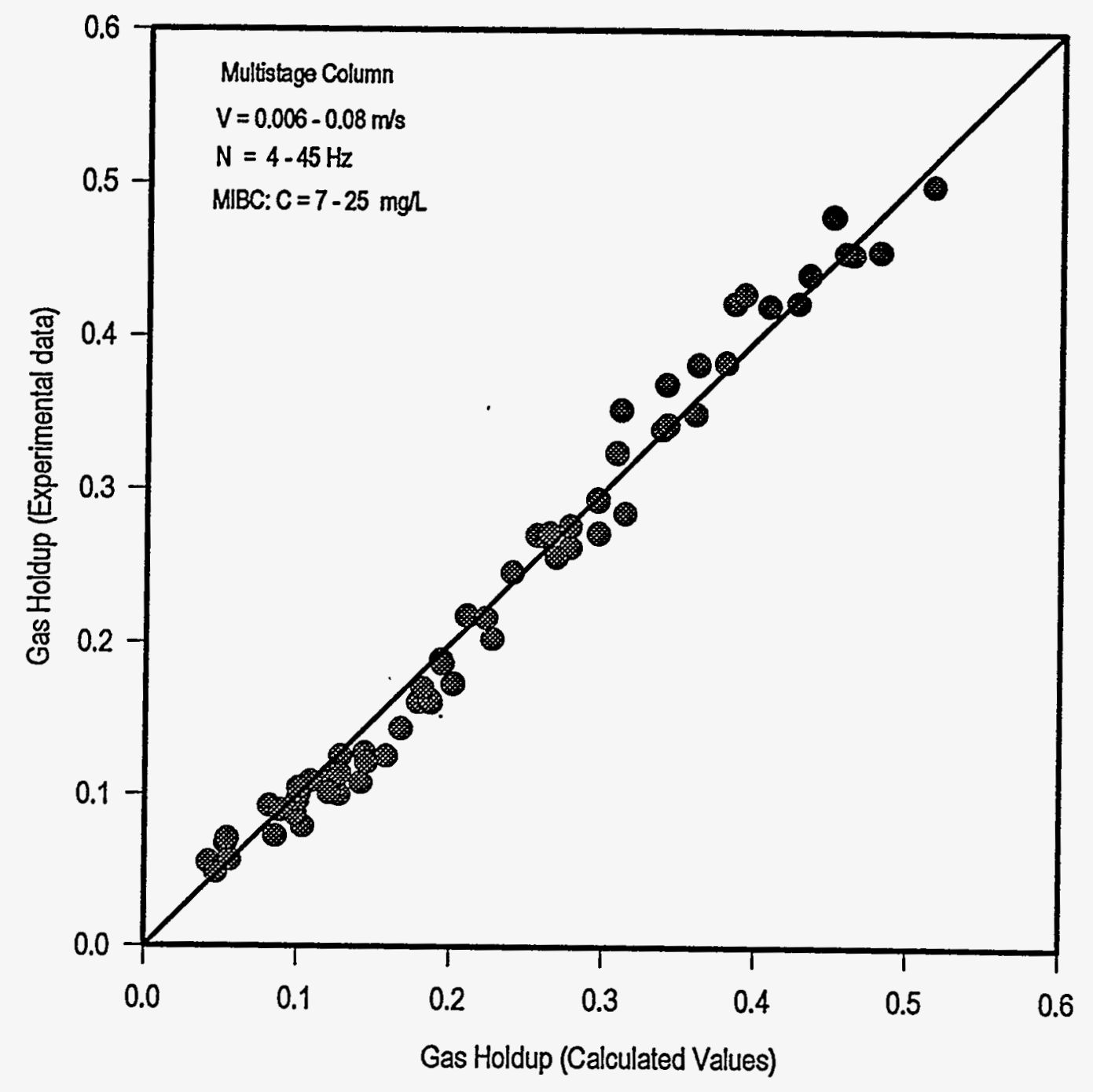

Figure 2.6.1 Comparison of Gas Holdup Between Calculated Values and Experimental Data 
Figure 2.6.2 depicts a comparison of bubble size between calculated values and experimental data obtained from multistage column. The relative deviation shown in the figure is approximately $\pm 10 \%$.

The bubble size data obtained from the multistage column and conventional column are listed in the Appendix.

\subsubsection{Specific Interfacial Area}

In the design and scale-up of the multistage column, the specific interfacial area of gas bubbles is one of the critical parameters. The specific interfacial area of gas bubbles is related to the magnitude of the gas holdup and the gas bubble Sauter mean diameter, $d_{a v}$. Mathematically, the specific interfacial area of gas bubbles can be expressed as:

$$
a=\frac{6 \Phi}{d_{a v}}
$$

Based on the data of the bubble Sauter mean diameter and the corresponding overall gas holdup, the specific interfacial area of gas bubble can be calculated. Table 2.6.1 shows a comparison of the specific interfacial area data between the multistage and conventional agitated column. The results indicate that the specific interfacial areas of gas bubbles in the multistage column are significantly greater than those in the conventional agitated column under the same operating conditions. The difference is primarily due to much larger gas holdup in the multistage column. As shown in Table 2.6.1, the effects of $\mathrm{N}, \mathrm{V}$ and MIBC dosage on $a$ are also very significant. 


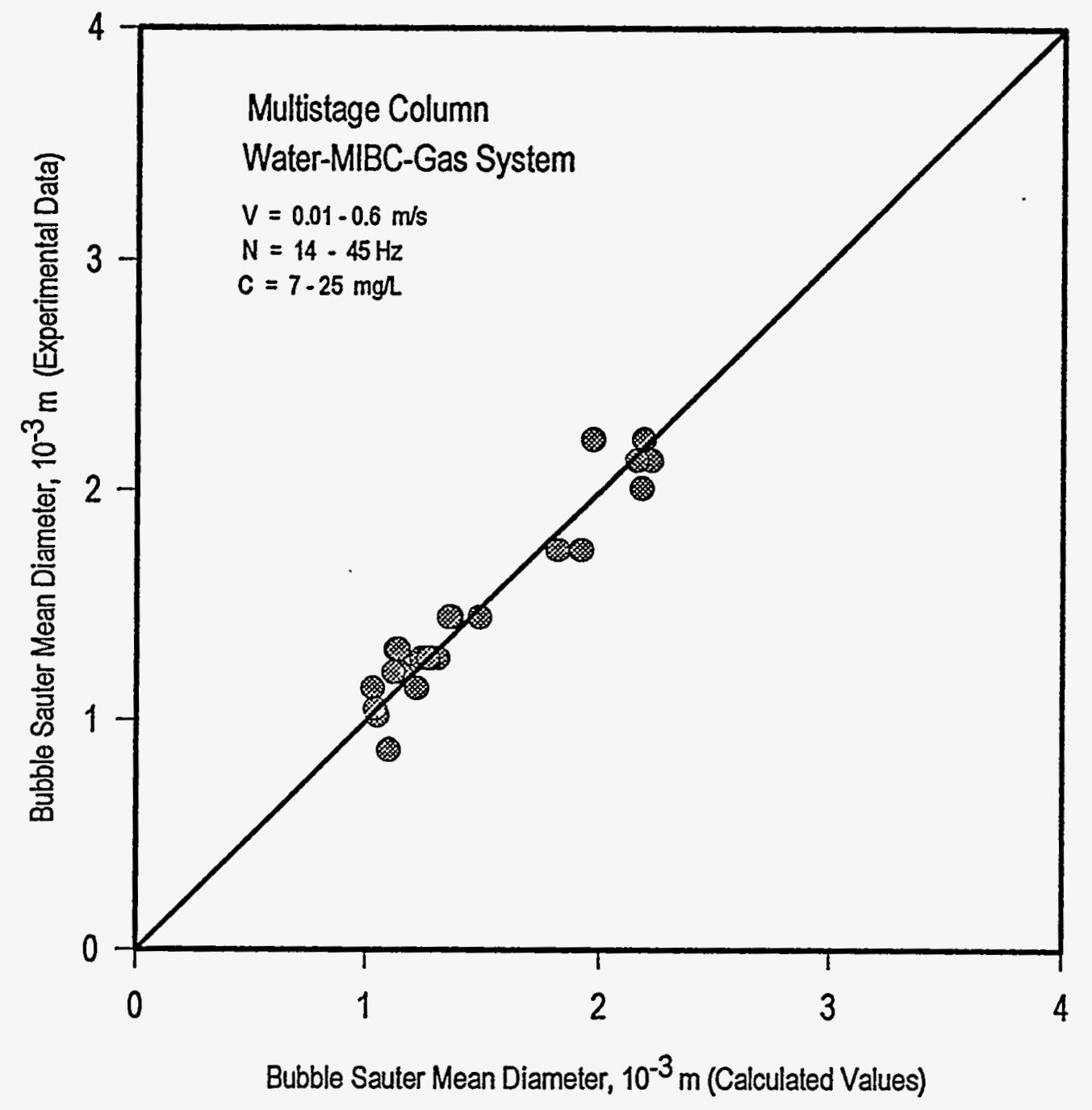

Figure 2.6.2 Comparison of Bubble Sizes Between Calculated Values and Experimental Data 
Table 2.6.1 Comparison of Specific Interfacial Area Data

\begin{tabular}{llllllll}
\hline MIBC, mg/L & 7.0 & 7.0 & 7.0 & 15.0 & 15.0 & 15.0 & 20.0 \\
$\mathrm{~N}, \mathrm{~Hz}$ & 14.7 & 30.0 & 30.0 & 21.7 & 21.7 & 45.0 & 21.7 \\
$\mathrm{~V}, \mathrm{~m} / \mathrm{s}$ & 0.04 & 0.04 & 0.04 & 0.03 & 0.05 & 0.03 & 0.02 \\
Multistage Column, $1 / \mathrm{m}$ & 518 & 595 & 603 & 968 & 1101 & 1543 & 940 \\
Conventional Column, $1 / \mathrm{m}$ & 373 & 490 & 481 & 614 & 657 & 996 & 845 \\
Percentage of Increase, \% & 38 & 21 & 25 & 58 & 68 & 55 & 11 \\
\hline
\end{tabular}

Based on Eq. (2.6.1) and using the multi-parameter regression program, the correlation for specific interfacial areas in the multistage column can be obtained as follows:

$$
\begin{aligned}
a= & 274.6-19.5 \mathrm{C}-16.1 \mathrm{~N}+4.66 \times 10^{3} \mathrm{~V}-1.37 \mathrm{C}^{2}-0.21 \mathrm{~N}^{2}-3.76 \times 10^{5} \mathrm{~V}^{2} \\
& +2.64 \mathrm{CN}+1.6 \times 10^{3} \mathrm{CV}+5.48 \times 10^{2} \mathrm{NV}
\end{aligned}
$$

Figure 2.6.3 presents a comparison of $a$ between calculated values and experimental data obtained from the multistage column. The relative deviation shown in the figure is approximately $\pm 10 \%$.

\subsubsection{Summary}

Experimental measurements of the overall gas holdup, gas bubble size and specific interfacial area were conducted to evaluate the roles of various operating parameters in the hydrodynamic behavior of the multistage column. Mathematical expressions developed using the multi-parameter regression method provide adequate correlations for the experimental data. In conclusion, the following points are noted: 


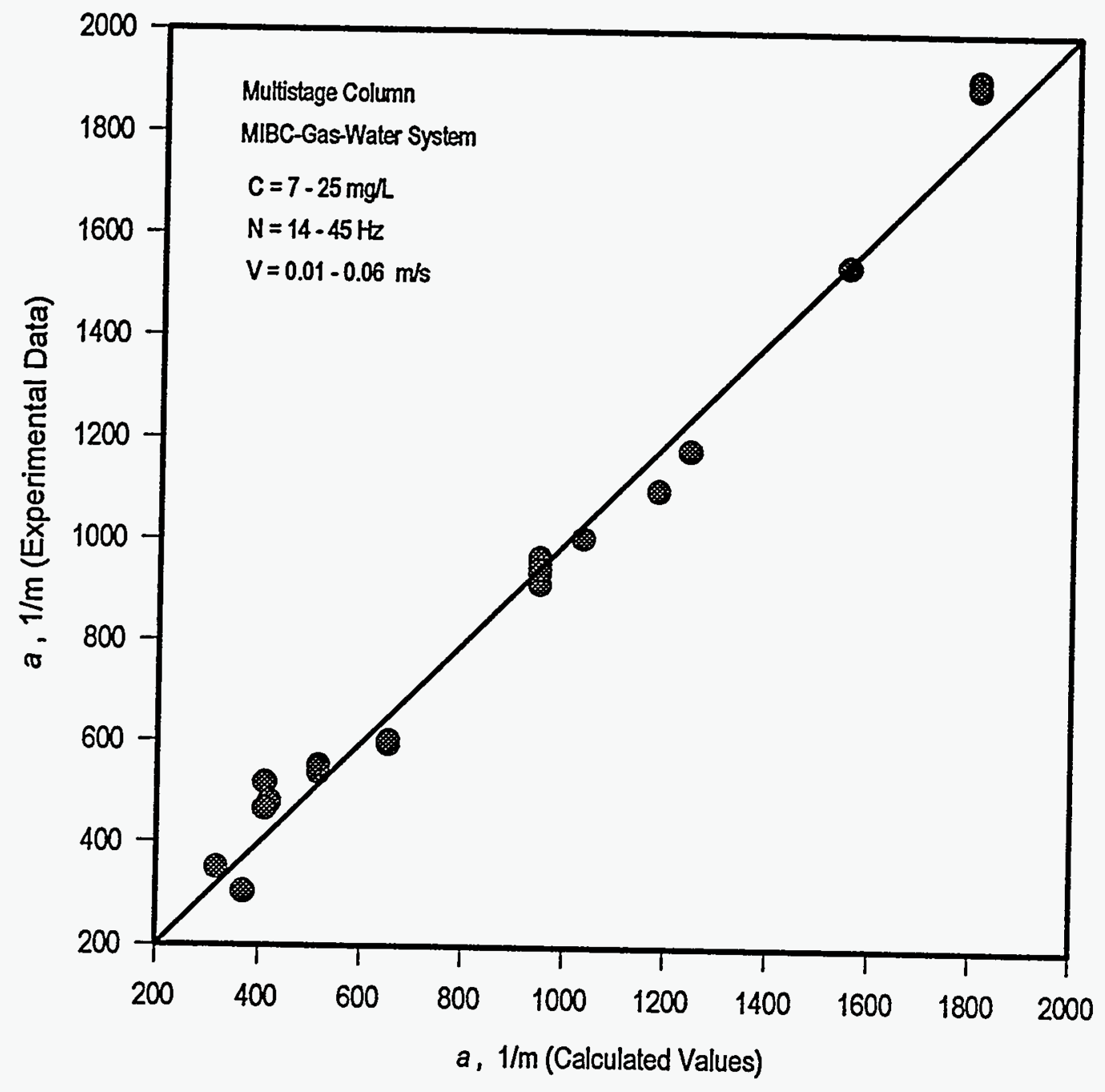

Figure 2.6.3 Comparison of Specific Area Between Calculated Values and Experimental Data 
1. The correlations for the gas holdup, bubble size and specific interfacial area under various operating range of $\mathrm{V}, \mathrm{N}$ and $\mathrm{C}$ have been developed. A comparison of calculated values with experimental data indicates that the relative deviations of these correlations are within $\pm 10 \sim 15 \%$.

2. The gas bubble Sauter diameters in the multistage column were found to range from 0.001 $\mathrm{m}$ to $0.002 \mathrm{~m}$, which are comparable to those in the conventional column.

3. The specific interfacial areas in the multistage column are significantly greater than those in the conventional column due to a higher gas holdup.

\subsection{WORK FORECAST}

In the next quarter, work for Tasks 3,5 , and 6 will be continued:

1. Conduct the investigation of mixing behavior in the multistage column:

a. Set up and calibrate the conductivity meter and auxiliary devices;

b. Carry out the measurements of mixing time under various operating conditions.

2. Initiate investigation of circulation flow characteristics around the contactor:

a. Define the circulation velocity and develop a measurement procedure;

b. Measure the circulation velocity around the contactor. 


\subsection{REFERENCES}

1. "A Study of Multistage/Multifunction Column for Fine Particle Separation", Quarterly Technical Progress Report, (July 8, 1994 - September 30, 1994), Chemical \& Petroleum Engineering Department, University of Pittsburgh, Submitted to U.S. Department of Energy, PETC, January 20, 1995.

2. “A Study of Multistage/Multifunction Column for Fine Particle Separation", Quarterly Technical Progress Report, (October 1, 1994 - December 31, 1994), Chemical \& Petroleum Engineering Department, University of Pittsburgh, Submitted to U.S. Department of Energy, PETC, February 2, 1995.

3. "A Study of Multistage/Multifunction Column for Fine Particle Separation", Quarterly Technical Progress Report, (January 1 - March 31, 1995), Chemical \& Petroleum Engineering Department, University of Pittsburgh, Submitted to U.S. Department of Energy, PETC, April 20, 1995.

4. "A Study of Multistage/Multifunction Column for Fine Particle Separation", Quarterly Technical Progress Report, (April 1, 1995 - June 30, 1995), Chemical \& Petroleum Engineering Department, University of Pittsburgh, Submitted to U.S. Department of Energy, PETC, July 20, 1995.

5. Mason, R. L., R. F. Gunst and J. L. Hess, <Statistical Design and Analysis of Experiments: with Applications to Engineering and Science>, John Wiley \& Sons, Inc., New York, 1989.

6. He, Da-Xin, "A Computer Program: Non-Linear Multi-Parameter Regression," (unpublished), September, 1995.

7. The Harmony User Manual, 2nd edition, Corporation of Canandaigua, New York, September 1993. 


\subsection{APPENDIX}

Table 5.1 Results of Sauter Mean Diameter of Bubbles and Interfacial Area

\begin{tabular}{|c|c|c|c|c|}
\hline \multirow[t]{2}{*}{ Run } & \multicolumn{2}{|c|}{ Multi-stage Column } & \multicolumn{2}{|c|}{ Conventional Column } \\
\hline & $\mathrm{d}_{32}\left(10^{-2} \mathrm{~m}\right)$ & $\mathrm{a}\left(10^{3} \mathrm{~m}^{-1}\right)$ & $\mathrm{d}_{32}\left(10^{-2} \mathrm{~m}\right)$ & $\mathrm{a}\left(10^{3} \mathrm{~m}^{-1}\right)$ \\
\hline 1 & 5.02 & 0.125 & 4.96 & 0.086 \\
\hline 2 & 1.92 & 0.288 & 1.98 & 0.215 \\
\hline 3 & 2.22 & 0.594 & 2.08 & 0.490 \\
\hline 4 & 1.37 & 0.477 & $1 / 30$ & 0.341 \\
\hline 5 & 1.97 & 0.518 & 2.09 & 0.373 \\
\hline 6 & 1.36 & 0.481 & 1.31 & 0.339 \\
\hline 7 & 2.19 & 0.466 & 2.27 & 0.344 \\
\hline 8 & 1.82 & 0.303 & 2.02 & 0.211 \\
\hline 9 & 2.19 & 0.603 & 2.12 & 0.481 \\
\hline 10 & 2.26 & 0.321 & 2.21 & 0.269 \\
\hline 11 & 1.03 & 0.350 & 0.99 & 0.236 \\
\hline 12 & 1.28 & 0.938 & 1.30 & 0.618 \\
\hline 13 & 1.31 & 0.916 & 1.29 & 0.623 \\
\hline 14 & 1.31 & 0.916 & 1.20 & 0.670 \\
\hline 15 & 1.31 & 0.916 & 1.31 & 0.614 \\
\hline 16 & 1.24 & 0.968 & 1.31 & 0.614 \\
\hline 17 & 1.25 & 0.960 & 1.35 & 0.596 \\
\hline 18 & 1.24 & 0.968 & 1.28 & 0.628 \\
\hline 19 & 1.31 & 0.916 & 1.31 & 0.614 \\
\hline 20 & 1.27 & 0.945 & 1.33 & 0.605 \\
\hline 21 & 2.18 & 1.101 & 2.28 & 0.658 \\
\hline 22 & 1.05 & 1.543 & 1.03 & 0.996 \\
\hline 23 & 1.25 & 0.960 & 1.67 & 0.467 \\
\hline 24 & 1.96 & 0.612 & 1.93 & 0.404 \\
\hline 25 & 1.04 & 0.940 & 0.98 & 0.845 \\
\hline 26 & 1.49 & 1.007 & 1.57 & 0.803 \\
\hline 27 & 1.13 & 1.912 & 1.11 & 1.784 \\
\hline 28 & 1.15 & 0.537 & 1.24 & 0.397 \\
\hline 29 & 1.14 & 1.895 & 1.15 & 1.722 \\
\hline 30 & 1.12 & 0.552 & 1.21 & 0.407 \\
\hline 31 & 1.10 & 0.889 & 0.97 & 0.854 \\
\hline 32 & 1.49 & 1.007 & 1.47 & 0.857 \\
\hline 33 & 1.22 & 1.180 & 1.18 & 1.017 \\
\hline
\end{tabular}


Table 5.2 Image Analysis of Bubble Sizes (Multistage Column : Run \#11)

\begin{tabular}{|c|c|c|c|c|c|}
\hline $\begin{array}{l}\text { Area } \\
\left(\mathrm{cm}^{2}\right)\end{array}$ & $x(\mathrm{~cm})$ & $\begin{array}{l}\text { tates } \\
\qquad y(\mathrm{~cm})\end{array}$ & $\begin{array}{c}d_{b} \\
(\mathrm{~mm})\end{array}$ & $\Sigma d_{b}^{2}$ & $\Sigma \mathrm{d}_{\mathrm{b}}{ }^{3}$ \\
\hline 0.005277 & 0.546232 & 0.893287 & 0.819707 & 0.671920 & 0.550778 \\
\hline 0.005907 & 0.436450 & 0.895927 & 0.867255 & 1.424051 & 1.203067 \\
\hline 0.012255 & 0.173566 & 0.841888 & 1.249137 & 2.984393 & 3.152147 \\
\hline 0.003295 & 0.667298 & 0.849463 & 0.647753 & 3.403978 & 3.423935 \\
\hline 0.002589 & 0.828093 & 0.834496 & 0.574127 & 3.733599 & 3.613179 \\
\hline 0.008330 & 0.337216 & 0.658176 & 1.029858 & 4.794207 & 4.705455 \\
\hline 0.001055 & 0.905802 & 0.790441 & 0.366518 & 4.928543 & 4.754692 \\
\hline 0.003324 & 0.625439 & 0.772499 & 0.650570 & 5.351784 & 5.030040 \\
\hline 0.005522 & 0.061030 & 0.751990 & 0.838526 & 6.054910 & 5.619630 \\
\hline 0.004802 & 0.712553 & 0.738281 & 0.781957 & 6.666367 & 6.097762 \\
\hline 0.002838 & 0.897593 & 0.712652 & 0.601095 & 7.027682 & 6.314947 \\
\hline 0.001325 & 0.544901 & 0.715053 & 0.410745 & 7.196394 & 6.384244 \\
\hline 0.008239 & 0.761473 & 0.641313 & 1.024248 & 8.245477 & 7.458765 \\
\hline 0.004238 & 0.591819 & 0.653705 & 0.734531 & 8.785012 & 7.855070 \\
\hline 0.002097 & 0.665756 & 0.631193 & 0.516686 & 9.051976 & 7.993007 \\
\hline 0.009038 & 0.054826 & 0.587420 & 1.072730 & 10.20272 & 9.227449 \\
\hline 0.005199 & 0.142281 & 0.563283 & 0.813587 & 10.86465 & 9.765982 \\
\hline 0.001651 & 0.572579 & 0.572936 & 0.458430 & 11.07481 & 9.862325 \\
\hline 0.001656 & 0.662916 & 0.575735 & 0.459227 & 11.28570 & 9.959171 \\
\hline 0.002147 & 0.865506 & 0.520355 & 0.522783 & 11.55900 & 10.10205 \\
\hline 0.007881 & 0.637175 & 0.467617 & 1.001744 & 12.56249 & 11.10729 \\
\hline 0.003579 & 0.221192 & 0.480032 & 0.675031 & 13.01816 & 11.41488 \\
\hline 0.004299 & 0.866646 & 0.435030 & 0.739822 & 13.56549 & 11.81981 \\
\hline 0.002516 & 0.944272 & 0.444040 & 0.566000 & 13.88585 & 12.00113 \\
\hline 0.001622 & 0.429717 & 0.433415 & 0.454423 & 14.09235 & 12.09497 \\
\hline 0.005894 & 0.713022 & 0.386023 & 0.866270 & 14.84277 & 12.74504 \\
\hline 0.001733 & 0.493339 & 0.408192 & 0.469724 & 15.06341 & 12.84868 \\
\hline 0.001522 & 0.051658 & 0.409052 & 0.440253 & 15.25724 & 12.93401 \\
\hline 0.003516 & 0.180600 & 0.367829 & 0.669045 & 15.70486 & 13.23349 \\
\hline 0.002141 & 0.394877 & 0.374773 & 0.522084 & 15.97743 & 13.37580 \\
\hline 0.001294 & 0.809679 & 0.372235 & 0.405969 & 16.14224 & 13.44270 \\
\hline 0.005557 & 0.531248 & 0.318722 & 0.841139 & 16.84975 & 14.03782 \\
\hline 0.001750 & 0.092793 & 0.329116 & 0.472055 & 17.07259 & 14.14301 \\
\hline 0.005624 & 0.341188 & 0.308883 & 0.846196 & 17.78864 & 14.74893 \\
\hline 0.007466 & 0.443433 & 0.296544 & 0.974980 & 18.73923 & 15.67573 \\
\hline 0.002526 & 0.779900 & 0.317321 & 0.567076 & 19.06080 & 15.85809 \\
\hline 0.003883 & 0.180914 & 0.276308 & 0.703159 & 19.55523 & 16.20576 \\
\hline 0.004180 & 0.618809 & 0.271004 & 0.729534 & 20.08745 & 16.59403 \\
\hline
\end{tabular}


Table 5.2 (Continued)

\begin{tabular}{|c|c|c|c|c|c|}
\hline 0.001538 & 0.269052 & 0.266457 & 0.442463 & 20.28323 & 16.68065 \\
\hline 0.002214 & 0.810078 & 0.260739 & 0.530882 & 20.56506 & 16.83027 \\
\hline 0.002583 & 0.042855 & 0.257292 & 0.573489 & 20.89395 & 17.01889 \\
\hline 0.003244 & 0.107743 & 0.228166 & 0.642652 & 21.30695 & 17.28430 \\
\hline 0.002386 & 0.552474 & 0.227631 & 0.551161 & 21.61073 & 17.45173 \\
\hline 0.003960 & 0.408526 & 0.198641 & 0.710059 & 22.11492 & 17.80973 \\
\hline 0.002342 & 0.217194 & 0.201875 & 0.546050 & 22.41309 & 17.97255 \\
\hline 0.003659 & 0.787543 & 0.189039 & 0.682573 & 22.87899 & 18.29057 \\
\hline 0.002302 & 0.626123 & 0.193140 & 0.541341 & 23.17204 & 18.44921 \\
\hline 0.005540 & 0.706766 & 0.171182 & 0.839834 & 23.87736 & 19.04156 \\
\hline 0.003763 & 0.865412 & 0.172909 & 0.692150 & 24.35644 & 19.37315 \\
\hline 0.001672 & 0.160257 & 0.162656 & 0.461346 & 24.56928 & 19.47134 \\
\hline 0.000200 & 0.057259 & 0.136871 & 0.506920 & 24.82624 & 19.60160 \\
\hline 0.002635 & 0.370463 & 0.111748 & 0.579199 & 25.16172 & 19.79591 \\
\hline 0.002457 & 0.120461 & 0.100799 & 0.559285 & 25.47451 & 19.97085 \\
\hline 0.002539 & 0.656992 & 0.100398 & 0.568579 & 25.79780 & 20.15466 \\
\hline 0.001760 & 0.824155 & 0.108482 & 0.473344 & 26.02185 & 20.26072 \\
\hline 0.005093 & 0.201750 & 0.083945 & 0.805304 & 26.67037 & 20.78297 \\
\hline 0.008565 & 0.706743 & 0.891153 & 1.044284 & 27.76089 & 21.92179 \\
\hline 0.005900 & 0.501282 & 0.876425 & 0.866833 & 28.51229 & 22.57313 \\
\hline 0.003470 & 0.158216 & 0.856005 & 0.664474 & 28.95382 & 22.86651 \\
\hline 0.001779 & 0.405848 & 0.843140 & 0.475913 & 29.18031 & 22.97430 \\
\hline 0.001970 & 0.227144 & 0.836812 & 0.501116 & 29.43143 & 23.10014 \\
\hline 0.001756 & 0.876079 & 0.823853 & 0.472829 & 29.65500 & 23.20585 \\
\hline 0.004395 & 0.100596 & 0.763470 & 0.748015 & 30.21452 & 23.62438 \\
\hline 0.000630 & 0.692873 & 0.790766 & 0.283216 & 30.29473 & 23.64710 \\
\hline 0.006150 & 0.255777 & 0.752664 & 0.884926 & 31.07783 & 24.34008 \\
\hline 0.002778 & 0.627736 & 0.763511 & 0.594776 & 31.43159 & 24.55049 \\
\hline 0.002656 & 0.518039 & 0.747894 & 0.581511 & 31.76974 & 24.74713 \\
\hline 0.002891 & 0.953364 & 0.728563 & 0.606747 & 32.13788 & 24.97050 \\
\hline 0.006338 & 0.425954 & 0.711170 & 0.898324 & 32.94487 & 25.69543 \\
\hline 0.001101 & 0.887006 & 0.736223 & 0.374414 & 33.08506 & 25.74792 \\
\hline 0.001756 & 0.574783 & 0.702713 & 0.472829 & 33.30862 & 25.85363 \\
\hline 0.003292 & 0.643158 & 0.687380 & 0.647377 & 33.72772 & 26.12494 \\
\hline 0.002962 & 0.136632 & 0.682469 & 0.614136 & 34.10488 & 26.35657 \\
\hline 0.001486 & 0.306815 & 0.674721 & 0.434961 & 34.29407 & 26.43886 \\
\hline 0.003619 & 0.739003 & 0.656855 & 0.678813 & 34.75486 & 26.75165 \\
\hline 0.002526 & 0.243208 & 0.648937 & 0.567076 & 35.07643 & 26.93401 \\
\hline 0.003311 & 0.810367 & 0.646384 & 0.649257 & 35.49797 & 27.20769 \\
\hline 0.002484 & 0.375754 & 0.617076 & 0.562327 & 35.81418 & 27.38551 \\
\hline 0.001844 & 0.179833 & 0.617225 & 0.484543 & 36.04896 & 27.49927 \\
\hline 0.001677 & 0.292090 & 0.591829 & 0.462137 & 36.26253 & 27.59797 \\
\hline 0.001564 & 0.485624 & 0.584895 & 0.446304 & 36.46172 & 27.68687 \\
\hline
\end{tabular}


Table 5.2 (Continued)

\begin{tabular}{|c|c|c|c|c|c|}
\hline 0.004521 & 0.716539 & 0.566599 & 0.758695 & 37.03734 & 28.12358 \\
\hline 0.011056 & 0.582988 & 0.537652 & 1.186473 & 38.44506 & 29.79380 \\
\hline 0.001639 & 0.043592 & 0.574435 & 0.456832 & 38.65375 & 29.88914 \\
\hline 0.002618 & 0.433260 & 0.538883 & 0.577302 & 38.98703 & 30.08155 \\
\hline 0.002759 & 0.230700 & 0.533209 & 0.592722 & 39.33835 & 30.28978 \\
\hline 0.002263 & 0.353054 & 0.523402 & 0.536819 & 39.62653 & 30.44448 \\
\hline 0.002914 & 0.099767 & 0.498150 & 0.609153 & 39.99759 & 30.67052 \\
\hline 0.002847 & 0.288525 & 0.462678 & 0.602108 & 40.36013 & 30.88880 \\
\hline 0.003056 & 0.174711 & 0.443059 & 0.623785 & 40.74924 & 31.13152 \\
\hline 0.001712 & 0.506380 & 0.436867 & 0.466861 & 40.96720 & 31.23328 \\
\hline 0.003753 & 0.426507 & 0.424239 & 0.691269 & 41.44505 & 31.56360 \\
\hline 0.007474 & 0.640773 & 0.393687 & 0.975480 & 42.39661 & 32.49183 \\
\hline 0.003569 & 0.093523 & 0.383335 & 0.674128 & 42.85106 & 32.79819 \\
\hline 0.002470 & 0.023795 & 0.379888 & 0.560808 & 43.16556 & 32.97457 \\
\hline 0.001959 & 0.391044 & 0.354644 & 0.499409 & 43.41497 & 33.09912 \\
\hline 0.002133 & 0.716739 & 0.348475 & 0.521149 & 43.68657 & 33.24067 \\
\hline 0.003010 & 0.269538 & 0.299223 & 0.619078 & 44.06983 & 33.47793 \\
\hline 0.002409 & 0.771503 & 0.298022 & 0.553808 & 44.37653 & 33.64779 \\
\hline 0.002305 & 0.052735 & 0.280756 & 0.541791 & 44.67007 & 33.80682 \\
\hline 0.002631 & 0.429600 & 0.270472 & 0.578778 & 45.00505 & 34.00070 \\
\hline 0.002614 & 0.226305 & 0.233890 & 0.576880 & 45.33784 & 34.19268 \\
\hline 0.004096 & 0.550910 & 0.221839 & 0.722145 & 45.85934 & 34.56928 \\
\hline 0.005262 & 0.690778 & 0.206599 & 0.818517 & 46.52931 & 35.11766 \\
\hline 0.002937 & 0.131728 & 0.208469 & 0.611550 & 46.90330 & 35.34638 \\
\hline 0.005821 & 0.879032 & 0.191610 & 0.860907 & 47.64446 & 35.98445 \\
\hline 0.002099 & 0.395453 & 0.152259 & 0.516921 & 47.91167 & 36.12257 \\
\hline 0.005532 & 0.508763 & 0.119690 & 0.839253 & 48.61601 & 36.71369 \\
\hline 0.002083 & 0.616387 & 0.114803 & 0.515032 & 48.88127 & 36.85031 \\
\hline 0.005582 & 0.752973 & 0.094263 & 0.843021 & 49.59195 & 37.44943 \\
\hline 0.003653 & 0.307959 & 0.079948 & 0.682038 & 50.05713 & 37.76670 \\
\hline 0.002304 & 0.674245 & 0.065531 & 0.541567 & 50.35042 & 37.92554 \\
\hline 0.000038 & 0.996582 & 0.036226 & 0.069829 & 50.35530 & 37.92588 \\
\hline 0.061100 & 0.993943 & 0.006059 & 2.788900 & 58.13327 & 59.61785 \\
\hline \multicolumn{5}{|c|}{ Average Size of Bubble (mm) } & 1.025538 \\
\hline
\end{tabular}


Table 5.3 Image Analysis of Bubble Size (Multistage Column: Run \#25)

\begin{tabular}{|c|c|c|c|c|c|}
\hline $\begin{array}{l}\text { Area } \\
\left(\mathrm{cm}^{2}\right)\end{array}$ & $\begin{array}{r}\text { Co } \\
x(\mathrm{~cm}) \\
\end{array}$ & $\begin{array}{l}\text { tes } \\
\mathrm{y}(\mathrm{cm})\end{array}$ & $\begin{array}{c}\mathrm{d}_{\mathrm{b}} \\
(\mathrm{mm})\end{array}$ & $\Sigma d_{b}^{2}$ & $\Sigma d_{b}^{3}$ \\
\hline 0.002512 & 0.536788 & 0.910661 & 0.565570 & 0.3198696 & 0.18090867 \\
\hline 0.001482 & 0.736669 & 0.916285 & 0.434400 & 0.5085726 & 0.26288117 \\
\hline 0.002091 & 0.927517 & 0.911370 & 0.515977 & 0.7748044 & 0.40025055 \\
\hline 0.001201 & 0.459884 & 0.912356 & 0.390978 & 0.9276682 & 0.46001696 \\
\hline 0.001294 & 0.676040 & 0.903397 & 0.405969 & 1.0924789 & 0.52692494 \\
\hline 0.002296 & 0.355981 & 0.881990 & 0.540666 & 1.3847981 & 0.68497187 \\
\hline 0.000655 & 0.601098 & 0.890854 & 0.288757 & 1.4681786 & 0.70904855 \\
\hline 0.000881 & 0.858775 & 0.884037 & 0.334887 & 1.5803278 & 0.74660586 \\
\hline 0.000708 & 0.804790 & 0.882629 & 0.300345 & 1.6705348 & 0.77369905 \\
\hline 0.002271 & 0.288739 & 0.856771 & 0.537727 & 1.9596849 & 0.92918282 \\
\hline 0.001921 & 0.138416 & 0.851498 & 0.494504 & 2.2042195 & 1.05010621 \\
\hline 0.000797 & 0.936054 & 0.861014 & 0.318468 & 2.3056414 & 1.08240586 \\
\hline 0.001810 & 0.675416 & 0.848411 & 0.479994 & 2.5360353 & 1.19299350 \\
\hline 0.002914 & 0.557244 & 0.838832 & 0.609153 & 2.9071031 & 1.41903069 \\
\hline 0.001181 & 0.412416 & 0.823243 & 0.387847 & 3.0575287 & 1.47737286 \\
\hline 0.002443 & 0.185651 & 0.800862 & 0.557756 & 3.3686206 & 1.65088624 \\
\hline 0.007583 & 0.801033 & 0.774473 & 0.982577 & 4.3340785 & 2.59952324 \\
\hline 0.001377 & 0.496291 & 0.783225 & 0.418682 & 4.5093730 & 2.67291587 \\
\hline 0.002717 & 0.110653 & 0.762933 & 0.588180 & 4.8553287 & 2.87640009 \\
\hline 0.002597 & 0.640486 & 0.757990 & 0.574975 & 5.1859254 & 3.06648498 \\
\hline 0.002621 & 0.549218 & 0.742195 & 0.577725 & 5.5196911 & 3.25930963 \\
\hline 0.001388 & 0.710989 & 0.750664 & 0.420425 & 5.6964485 & 3.33362295 \\
\hline 0.003271 & 0.361597 & 0.721878 & 0.645302 & 6.1128627 & 3.60233573 \\
\hline 0.000371 & 0.215661 & 0.729905 & 0.217480 & 6.1601604 & 3.61262204 \\
\hline 0.002570 & 0.469438 & 0.680060 & 0.571999 & 6.4873435 & 3.79977048 \\
\hline 0.021423 & 0.630820 & 0.627020 & 1.651563 & 9.2150042 & 8.30467445 \\
\hline 0.002191 & 0.765198 & 0.668517 & 0.528120 & 9.4939150 & 8.45197278 \\
\hline 0.002834 & 0.897151 & 0.663260 & 0.600690 & 9.8547434 & 8.66871878 \\
\hline 0.001783 & 0.175070 & 0.664807 & 0.476425 & 10.081724 & 8.77685782 \\
\hline 0.003619 & 0.285708 & 0.632650 & 0.678813 & 10.542510 & 9.08964560 \\
\hline 0.001574 & 0.161808 & 0.610693 & 0.447667 & 10.742916 & 9.17936036 \\
\hline 0.001718 & 0.486152 & 0.606034 & 0.467643 & 10.961606 & 9.28162945 \\
\hline 0.003230 & 0.860375 & 0.595615 & 0.641322 & 11.372901 & 9.54540187 \\
\hline 0.001564 & 0.742362 & 0.587503 & 0.446304 & 11.572087 & 9.63429967 \\
\hline 0.025094 & 0.285199 & 0.498552 & 1.787464 & 14.767116 & 15.3452996 \\
\hline 0.002338 & 0.124820 & 0.549450 & 0.545604 & 15.064800 & 15.5077168 \\
\hline 0.003244 & 0.806686 & 0.540952 & 0.642652 & 15.477801 & 15.7731334 \\
\hline 0.001961 & 0.703145 & 0.511405 & 0.499654 & 15.727456 & 15.8978742 \\
\hline 0.001028 & 0.942877 & 0.508105 & 0.361832 & 15.858378 & 15.9452459 \\
\hline 0.000804 & 0.643724 & 0.508572 & 0.319995 & 15.960775 & 15.9780126 \\
\hline
\end{tabular}


Table 5.3 (Continued)

\begin{tabular}{|c|c|c|c|c|c|}
\hline 0.001752 & 0.875477 & 0.497854 & 0.472313 & 16.183854 & 16.0833757 \\
\hline 0.002520 & 0.543742 & 0.483268 & 0.566431 & 16.504698 & 16.2651113 \\
\hline 0.001005 & 0.600514 & 0.494298 & 0.357766 & 16.632694 & 16.3109039 \\
\hline 0.001660 & 0.118841 & 0.439376 & 0.459757 & 16.844071 & 16.4080860 \\
\hline 0.002060 & 0.409082 & 0.440988 & 0.512183 & 17.106403 & 16.5424480 \\
\hline 0.002884 & 0.668971 & 0.425580 & 0.605943 & 17.473569 & 16.7649300 \\
\hline 0.001499 & 0.725450 & 0.412328 & 0.436917 & 17.664466 & 16.8483361 \\
\hline 0.000923 & 0.887277 & 0.417370 & 0.342801 & 17.781979 & 16.8886197 \\
\hline 0.001176 & 0.836316 & 0.410665 & 0.386904 & 17.931674 & 16.9465372 \\
\hline 0.001329 & 0.163478 & 0.395386 & 0.411339 & 18.100873 & 17.0161355 \\
\hline 0.001524 & 0.073360 & 0.383302 & 0.440531 & 18.294940 & 17.1016280 \\
\hline 0.002001 & 0.297939 & 0.381846 & 0.504751 & 18.549714 & 17.2302255 \\
\hline 0.001139 & 0.625041 & 0.387305 & 0.380871 & 18.694777 & 17.2854756 \\
\hline 0.001310 & 0.575414 & 0.383464 & 0.408364 & 18.861538 & 17.3535750 \\
\hline 0.000611 & 0.252826 & 0.364222 & 0.278878 & 18.939311 & 17.3752642 \\
\hline 0.001490 & 0.957656 & 0.359057 & 0.435521 & 19.128990 & 17.4578730 \\
\hline 0.003799 & 0.363694 & 0.339973 & 0.695489 & 19.612694 & 17.7942844 \\
\hline 0.000710 & 0.730003 & 0.339053 & 0.300750 & 19.703145 & 17.8214875 \\
\hline 0.000687 & 0.928516 & 0.325295 & 0.295846 & 19.790670 & 17.8473816 \\
\hline 0.001710 & 0.231857 & 0.304243 & 0.466601 & 20.008387 & 17.9489682 \\
\hline 0.002030 & 0.456094 & 0.305889 & 0.508361 & 20.266817 & 18.0803442 \\
\hline 0.000753 & 0.582694 & 0.313225 & 0.309539 & 20.362632 & 18.1100025 \\
\hline 0.007602 & 0.791294 & 0.264789 & 0.983817 & 21.330528 & 19.0622354 \\
\hline 0.001827 & 0.515460 & 0.283597 & 0.482274 & 21.563116 & 19.1744063 \\
\hline 0.002445 & 0.921845 & 0.267182 & 0.557975 & 21.874452 & 19.3481243 \\
\hline 0.002012 & 0.398574 & 0.258044 & 0.506198 & 22.130689 & 19.4778310 \\
\hline 0.001093 & 0.330423 & 0.264394 & 0.373110 & 22.269900 & 19.5297719 \\
\hline 0.000670 & 0.162359 & 0.263031 & 0.292114 & 22.355231 & 19.5546983 \\
\hline 0.001838 & 0.695366 & 0.242299 & 0.483788 & 22.589281 & 19.6679292 \\
\hline 0.001042 & 0.866960 & 0.245658 & 0.364182 & 22.721910 & 19.7162299 \\
\hline 0.001547 & 0.362538 & 0.212639 & 0.443839 & 22.918903 & 19.8036631 \\
\hline 0.002637 & 0.250454 & 0.207759 & 0.579410 & 23.254619 & 19.9981806 \\
\hline 0.002131 & 0.099528 & 0.208082 & 0.520915 & 23.525972 & 20.1395325 \\
\hline 0.000906 & 0.553952 & 0.209021 & 0.339586 & 23.641290 & 20.1786930 \\
\hline 0.000739 & 0.788193 & 0.181791 & 0.306770 & 23.735398 & 20.2075625 \\
\hline 0.003772 & 0.634022 & 0.133276 & 0.693031 & 24.215690 & 20.5404191 \\
\hline 0.004293 & 0.300573 & 0.130731 & 0.739328 & 24.762295 & 20.9445397 \\
\hline 0.003891 & 0.532444 & 0.129451 & 0.703852 & 25.257702 & 21.2932329 \\
\hline 0.000345 & 0.423689 & 0.142867 & 0.209486 & 25.301587 & 21.3024261 \\
\hline 0.018020 & 0.392697 & 0.806306 & 1.514737 & 27.596015 & 24.7778817 \\
\hline 0.019975 & 0.139288 & 0.755946 & 1.594787 & 30.139362 & 28.8339787 \\
\hline 0.001354 & 0.903811 & 0.793822 & 0.415173 & 30.311730 & 28.9055415 \\
\hline 0.000841 & 0.581937 & 0.751369 & 0.327153 & 30.418760 & 28.9405565 \\
\hline 0.001652 & 0.845662 & 0.727046 & 0.458695 & 30.629161 & 29.0370667 \\
\hline
\end{tabular}


Table 5.3 (Continued)

\begin{tabular}{|c|c|c|c|c|c|}
\hline 0.001310 & 0.328704 & 0.709181 & 0.408364 & 30.795922 & 29.1051661 \\
\hline 0.000820 & 0.429461 & 0.694083 & 0.323029 & 30.900270 & 29.1388733 \\
\hline 0.006978 & 0.610558 & 0.646265 & 0.942559 & 31.788687 & 29.9762582 \\
\hline 0.003368 & 0.043740 & 0.649307 & 0.654865 & 32.217535 & 30.2570963 \\
\hline 0.000714 & 0.365825 & 0.661624 & 0.301560 & 32.308474 & 30.2845197 \\
\hline 0.001128 & 0.300145 & 0.634674 & 0.378946 & 32.452073 & 30.3389362 \\
\hline 0.001191 & 0.149823 & 0.624131 & 0.389417 & 32.603719 & 30.3979894 \\
\hline 0.002654 & 0.791757 & 0.614458 & 0.581300 & 32.941629 & 30.5944167 \\
\hline 0.002650 & 0.883821 & 0.615428 & 0.580881 & 33.279051 & 30.7904189 \\
\hline 0.000885 & 0.421386 & 0.611072 & 0.335614 & 33.391688 & 30.8282214 \\
\hline 0.040255 & 0.286311 & 0.497507 & 2.263949 & 38.517152 & 42.4320073 \\
\hline 0.002551 & 0.661138 & 0.532298 & 0.569864 & 38.841896 & 42.6170676 \\
\hline 0.002461 & 0.577956 & 0.528197 & 0.559720 & 39.155183 & 42.7924207 \\
\hline 0.001032 & 0.124066 & 0.521854 & 0.362505 & 39.286593 & 42.8400573 \\
\hline 0.000758 & 0.818150 & 0.521406 & 0.310718 & 39.383139 & 42.8700559 \\
\hline 0.002342 & 0.506226 & 0.490679 & 0.546050 & 39.681310 & 43.0328723 \\
\hline 0.000948 & 0.631885 & 0.435702 & 0.347393 & 39.801992 & 43.0747965 \\
\hline 0.001634 & 0.752095 & 0.308839 & 0.456122 & 40.010039 & 43.1696915 \\
\hline 0.000494 & 0.889031 & 0.409015 & 0.250801 & 40.072940 & 43.1854671 \\
\hline 0.000860 & 0.564944 & 0.381091 & 0.330859 & 40.182408 & 43.2216853 \\
\hline 0.000777 & 0.623485 & 0.355718 & 0.314617 & 40.281392 & 43.2528273 \\
\hline 0.000754 & 0.155481 & 0.347712 & 0.309933 & 40.377450 & 43.2825989 \\
\hline 0.002669 & 0.065374 & 0.320449 & 0.582976 & 40.717311 & 43.4807294 \\
\hline 0.001203 & 0.341374 & 0.314789 & 0.391291 & 40.870419 & 43.5406393 \\
\hline 0.003276 & 0.473456 & 0.298882 & 0.645869 & 41.287565 & 43.8100610 \\
\hline 0.001765 & 0.899013 & 0.306636 & 0.474117 & 41.512352 & 43.9166361 \\
\hline 0.001084 & 0.202141 & 0.306250 & 0.371473 & 41.650344 & 43.9678966 \\
\hline 0.001281 & 0.280437 & 0.250574 & 0.403862 & 41.813449 & 44.0337683 \\
\hline 0.000772 & 0.353040 & 0.252786 & 0.313452 & 41.911701 & 44.0645658 \\
\hline 0.014917 & 0.616400 & 0.179999 & 1.378125 & 43.810929 & 46.6819381 \\
\hline 0.001153 & 0.126775 & 0.228831 & 0.383104 & 43.957698 & 46.7381659 \\
\hline 0.002631 & 0.493454 & 0.192446 & 0.578778 & 44.292682 & 46.9320474 \\
\hline 0.001210 & 0.253651 & 0.197494 & 0.392535 & 44.446765 & 46.9925306 \\
\hline 0.000559 & 0.785479 & 0.158178 & 0.266815 & 44.517956 & 47.0115253 \\
\hline 0.001264 & 0.190231 & 0.143374 & 0.401135 & 44.678865 & 47.0760717 \\
\hline 0.001036 & 0.395501 & 0.135609 & 0.363177 & 44.810763 & 47.1239738 \\
\hline 0.000561 & 0.830447 & 0.134246 & 0.267272 & 44.882197 & 47.1430662 \\
\hline 0.001681 & 0.688480 & 0.073973 & 0.462664 & 45.096255 & 47.2421033 \\
\hline 0.000944 & 0.290252 & 0.055103 & 0.346691 & 45.216450 & 47.2837737 \\
\hline \multirow[t]{2}{*}{0.001024} & 0.232672 & 0.050215 & 0.361157 & 45.346884 & 47.3308811 \\
\hline & & 18 tidg & Bubble & $(\mathrm{mm})$ & 1.04375156 \\
\hline
\end{tabular}


Table 5.4 Image Analysis of Bubble Size ( Conventional Column: Run \#13)

\begin{tabular}{|c|c|c|c|c|c|}
\hline $\begin{array}{l}\text { Area } \\
\left(\mathrm{cm}^{2}\right)\end{array}$ & $x(\mathrm{~cm})^{C}$ & $\begin{array}{l}\text { linates } \\
\qquad y(\mathrm{~cm})\end{array}$ & $\begin{array}{c}\mathrm{d}_{\mathrm{b}} \\
(\mathrm{mm})\end{array}$ & $\sum d_{b}^{2}$ & $\Sigma d_{b}^{3}$ \\
\hline 0.016485 & 0.586006 & 0.828279 & 1.448757 & 2.0988970 & 3.04079175 \\
\hline 0.000672 & 0.489196 & 0.882707 & 0.292531 & 2.1844716 & 3.06582504 \\
\hline 0.012443 & 0.137686 & 0.826781 & 1.258661 & 3.7686998 & 5.05983158 \\
\hline 0.001700 & 0.766053 & 0.817947 & 0.465292 & 3.9851963 & 5.16056566 \\
\hline 0.001995 & 0.465585 & 0.788745 & 0.504026 & 4.2392381 & 5.28860925 \\
\hline 0.011456 & 0.394472 & 0.728983 & 1.207756 & 5.6979121 & 7.05033117 \\
\hline 0.042515 & 0.181091 & 0.642571 & 2.326618 & 11.111064 & 19.6446684 \\
\hline 0.002505 & 0.495892 & 0.636603 & 0.564707 & 11.429958 & 19.8247503 \\
\hline 0.006210 & 0.569167 & 0.577107 & 0.889186 & 12.220609 & 20.5277859 \\
\hline 0.005118 & 0.745065 & 0.556063 & 0.807270 & 12.872294 & 21.0538713 \\
\hline 0.004745 & 0.918390 & 0.556650 & 0.777266 & 13.476437 & 21.5234515 \\
\hline 0.013764 & 0.388588 & 0.489495 & 1.323801 & 15.228886 & 23.8433443 \\
\hline 0.012421 & 0.240094 & 0.451120 & 1.257594 & 16.810427 & 25.8322808 \\
\hline 0.000515 & 0.641290 & 0.497211 & 0.256092 & 16.876010 & 25.8490760 \\
\hline 0.000620 & 0.591355 & 0.495153 & 0.281055 & 16.955002 & 25.8712772 \\
\hline 0.016596 & 0.094570 & 0.402810 & 1.453631 & 19.068045 & 28.9428612 \\
\hline 0.004216 & 0.623149 & 0.401191 & 0.732703 & 19.604899 & 29.3362155 \\
\hline 0.001446 & 0.433288 & 0.378916 & 0.429035 & 19.788970 & 29.4151884 \\
\hline 0.003175 & 0.607832 & 0.294691 & 0.635786 & 20.193194 & 29.6721886 \\
\hline 0.003586 & 0.042350 & 0.279942 & 0.675753 & 20.649836 & 29.9807660 \\
\hline 0.009187 & 0.462104 & 0.251919 & 1.081557 & 21.819601 & 31.2459324 \\
\hline 0.001779 & 0.689124 & 0.264573 & 0.475913 & 22.046093 & 31.3537232 \\
\hline 0.001476 & 0.782554 & 0.262072 & 0.433558 & 22.234065 & 31.4352199 \\
\hline 0.005294 & 0.226217 & 0.241727 & 0.821044 & 22.908180 & 31.9886976 \\
\hline 0.004393 & 0.331861 & 0.212734 & 0.747852 & 23.467463 & 32.4069588 \\
\hline 0.008140 & 0.883575 & 0.194010 & 1.018039 & 24.503867 & 33.4620590 \\
\hline 0.002242 & 0.620990 & 0.182969 & 0.534316 & 24.789360 & 33.6146026 \\
\hline 0.002616 & 0.172953 & 0.143861 & 0.577092 & 25.122395 & 33.8067943 \\
\hline 0.002325 & 0.801316 & 0.135070 & 0.544037 & 25.418371 & 33.9678161 \\
\hline 0.002330 & 0.561260 & 0.126200 & 0.544709 & 25.715079 & 34.1294357 \\
\hline 0.008262 & 0.417810 & 0.104915 & 1.025674 & 26.767087 & 35.2084529 \\
\hline 0.014752 & 0.654271 & 0.850033 & 1.370495 & 28.645344 & 37.7825956 \\
\hline 0.003292 & 0.193995 & 0.832095 & 0.647377 & 29.064441 & 38.0539093 \\
\hline 0.036428 & 0.361279 & 0.760459 & 2.153626 & 33.702547 & 48.0426548 \\
\hline 0.008546 & 0.095407 & 0.799384 & 1.043116 & 34.790638 & 49.1776604 \\
\hline 0.006627 & 0.735481 & 0.753756 & 0.918587 & 35.634439 & 49.9527648 \\
\hline 0.001363 & 0.912659 & 0.768763 & 0.416638 & 35.808026 & 50.0250878 \\
\hline 0.001074 & 0.654417 & 0.731384 & 0.369828 & 35.944799 & 50.0756701 \\
\hline 0.013892 & 0.120346 & 0.617183 & 1.329956 & 37.713583 & 52.4280760 \\
\hline 0.006495 & 0.812033 & 0.635531 & 0.909383 & 38.540561 & 53.1801159 \\
\hline
\end{tabular}


Table 5.4 (Continued)

\begin{tabular}{|c|c|c|c|c|c|}
\hline 0.000978 & 0.723982 & 0.657968 & 0.352963 & 38.665144 & 53.2240892 \\
\hline 0.004829 & 0.454319 & 0.614232 & 0.784137 & 39.280014 & 53.7062315 \\
\hline 0.006438 & 0.342443 & 0.586034 & 0.905353 & 40.099679 & 54.4483173 \\
\hline 0.000787 & 0.246815 & 0.605867 & 0.316548 & 40.199882 & 54.4800363 \\
\hline 0.001536 & 0.028968 & 0.569805 & 0.442188 & 40.395412 & 54.5664973 \\
\hline 0.003544 & 0.257071 & 0.517656 & 0.671772 & 40.846690 & 54.8696534 \\
\hline 0.001884 & 0.776590 & 0.526342 & 0.489797 & 41.086591 & 54.9871564 \\
\hline 0.002878 & 0.877061 & 0.493706 & 0.605339 & 41.453027 & 55.2089744 \\
\hline 0.000454 & 0.476719 & 0.502194 & 0.240377 & 41.510808 & 55.2228637 \\
\hline 0.001476 & 0.079865 & 0.487885 & 0.433558 & 41.698780 & 55.3043604 \\
\hline 0.001808 & 0.527997 & 0.486506 & 0.479739 & 41.928930 & 55.4147721 \\
\hline 0.000573 & 0.161093 & 0.466691 & 0.269995 & 42.001827 & 55.4344539 \\
\hline 0.051246 & 0.417650 & 0.344435 & 2.554387 & 48.526720 & 72.1015580 \\
\hline 0.002970 & 0.740386 & 0.414994 & 0.614929 & 48.904858 & 72.3340862 \\
\hline 0.003935 & 0.248353 & 0.404452 & 0.707824 & 49.405873 & 72.6887163 \\
\hline 0.003292 & 0.781780 & 0.334187 & 0.647377 & 49.824970 & 72.9600300 \\
\hline 0.004971 & 0.188236 & 0.323938 & 0.795558 & 50.457882 & 73.4635481 \\
\hline 0.007317 & 0.643578 & 0.292746 & 0.965178 & 51.389451 & 74.3626788 \\
\hline 0.001428 & 0.871269 & 0.316967 & 0.426471 & 51.571328 & 74.4402441 \\
\hline 0.000887 & 0.715408 & 0.297568 & 0.335977 & 51.684209 & 74.4781693 \\
\hline 0.003502 & 0.728268 & 0.210587 & 0.667769 & 52.130124 & 74.7759376 \\
\hline 0.001635 & 0.759450 & 0.147691 & 0.456298 & 52.338332 & 74.8709423 \\
\hline 0.001333 & 0.830097 & 0.148766 & 0.411931 & 52.508019 & 74.9408417 \\
\hline 0.001905 & 0.449251 & 0.136226 & 0.492528 & 52.750603 & 75.0603211 \\
\hline 0.000594 & 0.561631 & 0.120689 & 0.274916 & 52.826182 & 75.0810990 \\
\hline 0.003579 & 0.105040 & 0.099068 & 0.675031 & 53.281849 & 75.3886883 \\
\hline 0.001921 & 0.343807 & 0.106352 & 0.494504 & 53.526383 & 75.5096117 \\
\hline 0.002348 & 0.721051 & 0.092843 & 0.546719 & 53.825285 & 75.6730271 \\
\hline 0.001381 & 0.413578 & 0.089375 & 0.419264 & 54.001067 & 75.7467262 \\
\hline 0.001279 & 0.515630 & 0.084516 & 0.403559 & 54.163927 & 75.8124499 \\
\hline 0.001032 & 0.741752 & 0.890507 & 0.362505 & 54.295337 & 75.8600865 \\
\hline 0.003320 & 0.571042 & 0.862459 & 0.650195 & 54.718091 & 76.1349589 \\
\hline 0.008318 & 0.401531 & 0.816201 & 1.029115 & 55.777169 & 77.2248721 \\
\hline 0.004695 & 0.131365 & 0.779691 & 0.773178 & 56.374973 & 77.6870806 \\
\hline 0.015008 & 0.765132 & 0.727376 & 1.382363 & 58.285901 & 80.3286784 \\
\hline 0.001618 & 0.621089 & 0.773438 & 0.453886 & 58.491914 & 80.4221848 \\
\hline 0.021762 & 0.329123 & 0.664807 & 1.664575 & 61.262725 & 85.0344080 \\
\hline 0.001350 & 0.166705 & 0.692257 & 0.414585 & 61.434606 & 85.1056673 \\
\hline 0.000327 & 0.662515 & 0.683200 & 0.204182 & 61.476296 & 85.1141797 \\
\hline 0.002434 & 0.631884 & 0.630867 & 0.556663 & 61.786169 & 85.2866747 \\
\hline 0.001507 & 0.570701 & 0.633528 & 0.438032 & 61.978041 & 85.3707208 \\
\hline 0.000666 & 0.017163 & 0.629065 & 0.291279 & 62.062885 & 85.3954338 \\
\hline 0.009262 & 0.182449 & 0.569143 & 1.085943 & 63.242158 & 86.6760576 \\
\hline 0.001488 & 0.468426 & 0.593172 & 0.435241 & 63.431593 & 86.7585076 \\
\hline
\end{tabular}


Table 5.4 (Continued)

\begin{tabular}{llllll}
\hline 0.005953 & 0.681381 & 0.553071 & 0.870622 & 64.189575 & 87.4184236 \\
0.009702 & 0.562079 & 0.519810 & 1.111462 & 65.424923 & 88.7914657 \\
0.004140 & 0.370316 & 0.519844 & 0.726017 & 65.952024 & 89.1741499 \\
0.003424 & 0.911880 & 0.462723 & 0.660242 & 66.387943 & 89.4619617 \\
0.006001 & 0.658834 & 0.447757 & 0.874115 & 67.152020 & 90.1298533 \\
0.004663 & 0.471236 & 0.440309 & 0.770493 & 67.745679 & 90.5872636 \\
0.001967 & 0.023533 & 0.431700 & 0.500385 & 67.996064 & 90.7125525 \\
0.001053 & 0.097300 & 0.416259 & 0.366185 & 68.130156 & 90.7616547 \\
0.004986 & 0.627848 & 0.337922 & 0.796782 & 68.765017 & 91.2675007 \\
0.001099 & 0.068273 & 0.339060 & 0.374090 & 68.904960 & 91.3198519 \\
0.000553 & 0.930910 & 0.335008 & 0.265441 & 68.975419 & 91.3385546 \\
0.006304 & 0.449353 & 0.289732 & 0.895878 & 69.778017 & 92.0575846 \\
0.001721 & 0.319366 & 0.306137 & 0.468164 & 69.997195 & 92.1601960 \\
0.001607 & 0.537443 & 0.269801 & 0.452272 & 70.201745 & 92.2527081 \\
0.015537 & 0.086068 & 0.198043 & 1.406492 & 72.179964 & 95.0350574 \\
0.002518 & 0.811054 & 0.232503 & 0.566216 & 72.500564 & 95.2165865 \\
0.000331 & 0.387298 & 0.241498 & 0.205372 & 72.542742 & 95.2252486 \\
0.001953 & 0.250191 & 0.151670 & 0.498677 & 72.791421 & 95.3492591 \\
0.001415 & 0.310634 & 0.146633 & 0.424464 & 72.971591 & 95.4257347 \\
0.005404 & 0.611212 & 0.117326 & 0.829465 & 73.659602 & 95.9964158 \\
0.001396 & 0.531973 & 0.124771 & 0.421582 & 73.837334 & 96.0713443 \\
\hline & & Average Size of Bubble & $(\mathrm{mm})$ & \\
\hline
\end{tabular}


Table 5.4 Image Analysis of Bubble Size ( Conventional Column: Run \#24)

\begin{tabular}{|c|c|c|c|c|c|}
\hline $\begin{array}{l}\text { Area } \\
\left(\mathrm{cm}^{2}\right)\end{array}$ & $x(\mathrm{~cm})$ & $\begin{array}{l}\text { dinates } \\
\mathrm{y}(\mathrm{cm})\end{array}$ & $\begin{array}{c}\mathrm{d}_{\mathrm{b}} \\
(\mathrm{mm})\end{array}$ & $\Sigma d_{b}^{2}$ & $\Sigma \mathrm{d}_{b}^{3}$ \\
\hline 0.004733 & 0.307330 & 0.866147 & 0.776325 & 0.60268023 & 0.46787563 \\
\hline 0.002629 & 0.687285 & 0.770105 & 0.578568 & 0.93742124 & 0.66154608 \\
\hline 0.005746 & 0.307199 & 0.741204 & 0.855366 & 1.66907298 & 1.28737643 \\
\hline 0.003772 & 0.077117 & 0.617024 & 0.693031 & 2.14936435 & 1.62023303 \\
\hline 0.001465 & 0.875053 & 0.589648 & 0.431867 & 2.33587355 & 1.70078022 \\
\hline 0.010016 & 0.362517 & 0.503399 & 1.129304 & 3.61120107 & 3.14101268 \\
\hline 0.020207 & 0.775257 & 0.466457 & 1.604010 & 6.18404867 & 7.26788557 \\
\hline 0.003945 & 0.507707 & 0.396893 & 0.708684 & 6.68628177 & 7.62381016 \\
\hline 0.003699 & 0.340438 & 0.342228 & 0.686313 & 7.15730777 & 7.94708159 \\
\hline 0.033496 & 0.855638 & 0.266225 & 2.065150 & 11.4221505 & 16.7546196 \\
\hline 0.002891 & 0.603279 & 0.34017 & 0.606747 & 11.7902924 & 16.9779886 \\
\hline 0.002068 & 0.512460 & 0.310885 & 0.513135 & 12.0535996 & 17.1131007 \\
\hline 0.009723 & 0.257089 & 0.245623 & 1.112668 & 13.2916288 & 18.4906157 \\
\hline 0.003573 & 0.421165 & 0.213773 & 0.674489 & 13.7465649 & 18.7974653 \\
\hline 0.018191 & 0.279796 & 0.106065 & 1.521882 & 16.0626892 & 22.3223329 \\
\hline 0.00278 & 0.852742 & 0.104195 & 0.594980 & 16.4166905 & 22.5329566 \\
\hline 0.033777 & 0.309995 & 0.805554 & 2.073806 & 20.7173622 & 31.4517157 \\
\hline 0.119946 & 0.754276 & 0.730508 & 3.907940 & 35.9893595 & 91.1337696 \\
\hline 0.007093 & 0.475415 & 0.737079 & 0.950287 & 36.8924046 & 91.9919214 \\
\hline 0.046791 & 0.556291 & 0.430883 & 2.440812 & 42.8499682 & 106.533214 \\
\hline 0.001867 & 0.162213 & 0.397996 & 0.487552 & 43.0876756 & 106.649109 \\
\hline 0.003958 & 0.738329 & 0.294178 & 0.709888 & 43.5916161 & 107.00685 \\
\hline 0.054894 & 0.401207 & 0.193688 & 2.643735 & 50.5809492 & 125.484793 \\
\hline 0.002937 & 0.840077 & 0.198882 & 0.611550 & 50.9549429 & 125.713509 \\
\hline 0.010650 & 0.142018 & 0.173775 & 1.164485 & 52.3109684 & 127.29258 \\
\hline 0.003112 & 0.755545 & 0.867488 & 0.629427 & 52.7071470 & 127.541946 \\
\hline 0.002049 & 0.907553 & 0.823902 & 0.510753 & 52.9680159 & 127.675185 \\
\hline 0.001306 & 0.285724 & 0.824842 & 0.407767 & 53.1342895 & 127.742986 \\
\hline 0.003569 & 0.506760 & 0.812099 & 0.674128 & 53.5887379 & 128.049343 \\
\hline 0.005746 & 0.711178 & 0.709199 & 0.855366 & 54.3203897 & 128.675173 \\
\hline 0.072980 & 0.215542 & 0.502840 & 3.048286 & 63.6124399 & 157.000004 \\
\hline 0.021331 & 0.865913 & 0.525317 & 1.648017 & 66.3283996 & 161.475951 \\
\hline 0.004138 & 0.525035 & 0.526405 & 0.725850 & 66.8552572 & 161.858371 \\
\hline 0.011236 & 0.654718 & 0.454947 & 1.196092 & 68.2858944 & 163.569545 \\
\hline 0.002064 & 0.722264 & 0.269600 & 0.512659 & 68.5487140 & 163.704282 \\
\hline 0.003653 & 0.364545 & 0.260695 & 0.682038 & 69.0138895 & 164.021549 \\
\hline 0.012033 & 0.543420 & 0.237388 & 1.237760 & 70.5459402 & 165.917861 \\
\hline 0.002951 & 0.672453 & 0.112340 & 0.612944 & 70.9216401 & 166.148144 \\
\hline 0.001042 & 0.259305 & 0.054805 & 0.364182 & 71.0542683 & 166.196445 \\
\hline 0.015219 & 0.233834 & 0.704129 & 1.392028 & 72.9920114 & 168.893838 \\
\hline
\end{tabular}


Table 5.5 (Continued)

\begin{tabular}{|c|c|c|c|c|c|}
\hline 0.002348 & 0.593063 & 0.722065 & 0.546719 & 73.2909132 & 169.057253 \\
\hline 0.032983 & 0.868021 & 0.650092 & 2.049268 & 77.4904133 & 177.663155 \\
\hline 0.058463 & 0.545006 & 0.530413 & 2.728329 & 84.9341910 & 197.972228 \\
\hline 0.001995 & 0.161777 & 0.562202 & 0.504026 & 85.1882328 & 198.100271 \\
\hline 0.002122 & 0.296793 & 0.539128 & 0.519743 & 85.4583659 & 198.240671 \\
\hline 0.001589 & 0.145803 & 0.438367 & 0.449840 & 85.6607218 & 198.331699 \\
\hline 0.003966 & 0.723596 & 0.383653 & 0.710573 & 86.1656364 & 198.690478 \\
\hline 0.012877 & 0.098440 & 0.326962 & 1.280459 & 87.8052122 & 200.789888 \\
\hline 0.001649 & 0.558575 & 0.316160 & 0.458164 & 88.0151261 & 200.886063 \\
\hline 0.010072 & 0.847134 & 0.220599 & 1.132434 & 89.2975328 & 202.338304 \\
\hline 0.002141 & 0.215080 & 0.240278 & 0.522084 & 89.5701041 & 202.480609 \\
\hline 0.002147 & 0.100101 & 0.211597 & 0.522783 & 89.8434062 & 202.623486 \\
\hline 0.006863 & 0.214224 & 0.106720 & 0.934767 & 90.7171947 & 203.440275 \\
\hline 0.005924 & 0.580235 & 0.101866 & 0.868519 & 91.4715203 & 204.095421 \\
\hline 0.002803 & 0.794026 & 0.804412 & 0.597434 & 91.8284475 & 204.308661 \\
\hline 0.002574 & 0.707819 & 0.699011 & 0.572425 & 92.1561182 & 204.496228 \\
\hline 0.005674 & 0.535101 & 0.563542 & 0.849933 & 92.8785046 & 205.110209 \\
\hline 0.000484 & 0.405359 & 0.580452 & 0.248359 & 92.9401867 & 205.125528 \\
\hline 0.005241 & 0.769125 & 0.535349 & 0.816877 & 93.6074749 & 205.67062 \\
\hline 0.002547 & 0.442324 & 0.523263 & 0.569436 & 93.9317321 & 205.855264 \\
\hline 0.002562 & 0.320667 & 0.500767 & 0.571146 & 94.2579398 & 206.041576 \\
\hline 0.005942 & 0.644782 & 0.475825 & 0.869781 & 95.0144592 & 206.699583 \\
\hline 0.004329 & 0.266147 & 0.428191 & 0.742454 & 95.5656968 & 207.108851 \\
\hline 0.001988 & 0.515080 & 0.407889 & 0.503057 & 95.8187633 & 207.236158 \\
\hline 0.005128 & 0.363145 & 0.388549 & 0.808024 & 96.4716664 & 207.763719 \\
\hline 0.007271 & 0.084155 & 0.335558 & 0.962143 & 97.3973853 & 208.654393 \\
\hline 0.004015 & 0.855088 & 0.241445 & 0.715020 & 97.9086393 & 209.01995 \\
\hline 0.006564 & 0.361361 & 0.085728 & 0.914197 & 98.744395 & 209.783995 \\
\hline 0.009136 & 0.828687 & 0.853501 & 1.078509 & 99.9075768 & 211.038497 \\
\hline 0.002656 & 0.636450 & 0.856945 & 0.581511 & 100.245731 & 211.235138 \\
\hline 0.003632 & 0.507385 & 0.792617 & 0.680069 & 100.708225 & 211.549666 \\
\hline 0.007343 & 0.187759 & 0.748784 & 0.966945 & 101.643208 & 212.453743 \\
\hline 0.003410 & 0.636629 & 0.760477 & 0.658948 & 102.077421 & 212.739867 \\
\hline 0.002912 & 0.406376 & 0.751793 & 0.608953 & 102.448245 & 212.965681 \\
\hline 0.001139 & 0.866162 & 0.720209 & 0.380871 & 102.593307 & 213.020931 \\
\hline 0.013360 & 0.080055 & 0.656138 & 1.304228 & 104.294317 & 215.239435 \\
\hline 0.009903 & 0.327262 & 0.651409 & 1.122919 & 105.555263 & 216.655375 \\
\hline 0.020939 & 0.825198 & 0.629028 & 1.632784 & 108.221248 & 221.008354 \\
\hline 0.005702 & 0.646817 & 0.625931 & 0.852082 & 108.947293 & 221.627003 \\
\hline 0.008661 & 0.208035 & 0.584278 & 1.050105 & 110.050012 & 222.784974 \\
\hline 0.001597 & 0.311494 & 0.545902 & 0.450923 & 110.253343 & 222.876661 \\
\hline 0.011453 & 0.049845 & 0.493877 & 1.207550 & 111.711521 & 224.637483 \\
\hline 0.001294 & 0.426625 & 0.521515 & 0.405969 & 111.876332 & 224.704391 \\
\hline 0.003715 & 0.833173 & 0.504468 & 0.687733 & 112.349308 & 225.029673 \\
\hline
\end{tabular}


Table 5.5 (Continued)

\begin{tabular}{llllll}
\hline 0.009017 & 0.693911 & 0.469583 & 1.071478 & 113.497374 & 226.259801 \\
0.007047 & 0.275639 & 0.460436 & 0.947203 & 114.394567 & 227.109625 \\
0.000643 & 0.598816 & 0.461846 & 0.286213 & 114.476485 & 227.133071 \\
0.004494 & 0.826944 & 0.389438 & 0.756442 & 115.048690 & 227.565911 \\
0.013283 & 0.107343 & 0.360790 & 1.300483 & 116.739947 & 229.765362 \\
0.002989 & 0.255903 & 0.335390 & 0.616909 & 117.120523 & 230.000143 \\
0.006037 & 0.448924 & 0.312241 & 0.876761 & 117.889233 & 230.674117 \\
0.003196 & 0.342864 & 0.299935 & 0.637893 & 118.296140 & 230.93368 \\
0.039710 & 0.865835 & 0.165661 & 2.248551 & 123.352122 & 242.302316 \\
0.002214 & 0.175016 & 0.245272 & 0.530882 & 123.633958 & 242.451937 \\
0.013823 & 0.689999 & 0.186528 & 1.326654 & 125.393969 & 244.786864 \\
0.003684 & 0.283534 & 0.188534 & 0.684892 & 125.863046 & 245.108131 \\
0.003743 & 0.127987 & 0.179682 & 0.690387 & 126.339681 & 245.437193 \\
0.002143 & 0.487822 & 0.178893 & 0.522316 & 126.612495 & 245.579689 \\
0.005574 & 0.213279 & 0.112636 & 0.842442 & 127.322204 & 246.177577 \\
0.000774 & 0.556663 & 0.105712 & 0.313841 & 127.420700 & 246.208490 \\
\hline
\end{tabular}

